Vol. 37 (1988) [149-160]

\title{
NONEXPANSIVE PROJECTIONS ONTO TWO-DIMENSIONAL SUBSPACES OF BANACH SPACES
}

\author{
Bruce Calvert and Simon Fitzpatrick
}

\begin{abstract}
We show that if a three dimensional normed space $X$ has two linearly independent smooth points $e$ and $f$ such that every two-dimensional subspace containing $e$ or $f$ is the range of a nonexpansive projection then $X$ is isometrically isomorphic to $\ell_{p}(3)$ for some $p$, $1<p \leqslant \infty$. This leads to a characterisation of the Banach spaces $c_{0}$ and $\ell_{p}, 1<p \leqslant \infty$, and a characterisation of real Hilbert spaces.
\end{abstract}

\section{INTRODUCTION}

In 1969, Ando [1] showed that a real three dimensional Banach lattice is isometrically isomorphic to $\ell_{p}(3)$ for some $p \in[1, \infty]$ if and only if all sublattices are ranges of positive nonexpansive projections. This and other results on characterising $L_{p}$ spaces can be found in the books [6] and [8]. In recent work [3] and [4] we characterised $\ell_{p}(3)$ by only requiring sublattices through two of the coordinate axes to be ranges of nonexpansive projections. This allowed us to characterise the Banach lattices $\ell_{p}(n)$, $c_{0}$ and $\ell_{p}$ by requiring planes through $R e_{i}$ to be ranges of nonexpansive projections for certain disjoint elements $e_{i}$.

In this work we show that those results generalise to Banach spaces which are not endowed with lattice structure and to $e_{i}$ which are not necessarily orthogonal. In Theorem $\mathrm{A}$ we take two linearly independent smooth points $e$ and $f$ in a threedimensional normed space $X$ such that every two-dimensional subspace which intersects $\{e, f\}$ is the range of a nonexpansive projection and conclude that $X$ is $\ell_{p}(3)$. If $e$ and $f$ are not orthogonal then we have $p=2$.

We extend this result to higher dimensions in Theorems B and C. This yields a characterisation of $\ell_{p}$ and $c_{0}$ which requires only a small number of planes to be ranges of nonexpansive projections. In Theorem $D$ we use this to characterise Hilbert spaces.

Let $X$ be a Banach space. Recall that duality mapping $J$ from $X$ to subsets of $X^{\star}$ is defined by $x^{\star} \in J x$ provided $x^{\star}(x)=\|x\|^{2}=\left\|x^{\star}\right\|^{2}$. The norm is smooth at $x$, or $x$ is a smooth point, provided $J x$ is a singleton. By projection we mean a linear map $P: X \rightarrow X$ such that $P^{2}=P$. A point $x$ is orthogonal to a point $y$ provided $\|x+t y\| \geqslant\|x\|$ for all $t \in \mathbf{R}$.

Received 2 March 1987

Copyright Clearance Centre, Inc. Serial-fee code: 0004-9729/88 \$A2.00+0.00. 


\section{ThrEe-DIMENSIONAL NORMED SPACES}

The following result is basically by Blaschke [2] and appears in a form like this in Ando [1]. We give a different proof.

LEMMA 1. Let $X$ be a real three-dimensional normed space with basis $\left\{e_{1}, e_{2}, e_{3}\right\}$ where $e_{1}$ is a unit vector. Suppose every two-dimensional subspace which contains $e_{1}$ is the range of a nonexpansive projection along a vector in $\operatorname{span}\left\{e_{2}, e_{3}\right\}$. Then there is a function $F: \mathbf{R}^{2} \rightarrow \mathbf{R}$ such that

$$
\left\|x_{1} e_{1}+x_{2} e_{2}+x_{3} e_{3}\right\|=F\left(x_{1},\left\|x_{2} e_{2}+x_{3} e_{3}\right\|\right) \quad \text { for all } x_{i} \in \mathbf{R}
$$

Proof: Let $y(t), 0 \leqslant t \leqslant T$, be a parametrization of the unit circle $\|y(t)\|=1$ in $\operatorname{span}\left\{e_{2}, e_{3}\right\}$, such that

$$
\lim _{h \rightarrow 0_{+}} \frac{y(t+h)-y(t)}{h}=p(t), \quad\|p(t)\|=1,
$$

and $y(0)=y(T)=\left\|e_{2}\right\|^{-1} e_{2}$. Then from the existance of a nonexpansive projection onto $\operatorname{span}\left\{e_{1}, y(t+h)\right\}$ along a vector $u(t+h)$ in $\operatorname{span}\left\{e_{2}, e_{3}\right\}$ for each $t$ we see by taking the limit as $h \rightarrow 0_{+}$that the projection along $p(t)$ is nonexpansive, so that $\left\|x_{1} e_{1}+y(t)+s p(t)\right\| \geqslant\left\|x_{1} e_{1}+y(t)\right\|$ for all $x_{1}, s$ and $t$. Now for $h>0$,

$$
\begin{aligned}
\left\|x_{1} e_{1}+y(t+h)\right\| & =\left\|x_{1} e_{1}+y(t)+y(t+h)-y(t)\right\| \\
& \geqslant\left\|x_{1} e_{1}+y(t)+h p(t)\right\|-\|y(t+h)-y(t)-h p(t)\| \\
& \geqslant\left\|x_{1} e_{1}+y(t)\right\|-\|y(t+h)-y(t)-h p(t)\|
\end{aligned}
$$

so that the right-hand derivative of $\left\|x_{1} e_{1}+y(t)\right\|$,

$$
\lim _{h \rightarrow 0_{+}} \frac{\left\|x_{1} e_{1}+y(t+h)\right\|-\left\|x_{1} e_{1}+y(t)\right\|}{h} \geqslant 0 .
$$

Since $y(0)=y(T)$ we see that $\left\|x_{1} e_{1}+y(t)\right\|=F\left(x_{1}, 1\right)$ does not depend on $t$. The result follows by homogeneity of the norm.

Now we introduce some standing assumptions for this Section.

Standing assumptions. Let $X$ be a real three-dimensional normed space with two linearly independent smooth points of norm $1, e$ and $f$, such that every twodimensional subspace which intersects $\{e, f\}$ is the range of a nonexpansive projection. Let $e_{1}=e, f_{1}=f$, choose unit vectors $e_{2}$ and $f_{2}$ in $\operatorname{span}\{e, f\}$ such that $J e\left(e_{2}\right)=0=J f\left(f_{2}\right)$ and let $e_{3}$ be a unit vector such that $J e\left(e_{3}\right)=0=J f\left(e_{3}\right)$, and $e_{3} \notin \operatorname{span}\{e, f\}$. 
Proposition 2. For all numbers $x_{1}, x_{2}$ and $x_{3}$ we have

$$
\left\|\sum_{i=1}^{3} x_{i} e_{i}\right\|=\left\|\sum_{i=1}^{3}\left|x_{i}\right| e_{i}\right\|
$$

Proof: For any two-dimensional subspace $M$ containing $e$, the nonexpansive projection onto $M$ is in a direction $p$ tangent to $e$ so that $J e(p)=0$. Thus all such $p$ are in $\operatorname{span}\left\{e_{2}, e_{3}\right\}$.

By Lemma 1 we have for all $x_{i}$,

$$
\left\|x_{1} e_{1}+x_{2} e_{2}+x_{3} e_{3}\right\|=\left\|x_{1} e_{1} \pm\right\| x_{2} e_{2}+x_{3} e_{3}\left\|e_{3}\right\| \text {. }
$$

Now to show (1) we only have to show

$$
\left\|x_{2} e_{2}+x_{3} e_{3}\right\|=\left\|x_{2} e_{2}-x_{3} e_{3}\right\|
$$

for all $x_{3}$ and $x_{2}$.

Considering $f$ instead of $e$ we have

$$
\left\|y_{1} f_{1}+y_{2} f_{2}+y_{3} f_{3}\right\|=\left\|y_{1} f_{1} \pm\right\| y_{2} f_{2}+y_{3} f_{3}\left\|e_{3}\right\|
$$

for all $y_{1}, y_{2}$ and $y_{3}$.

Now let

$$
f_{1}=\alpha e_{1}+\beta e_{2}, \text { so } \beta \neq 0, \text { and } f_{2}=\gamma e_{1}+\delta e_{2} \text {. }
$$

Thus for any $t$,

$$
\begin{aligned}
\left\|f_{1}+t f_{1}\right\| & =\left\|f_{1}+t e_{3}\right\| \quad \text { by }(4) \\
& =\left\|\alpha e_{1}+\beta e_{2}+t e_{3}\right\| \quad \text { by }(5) \\
& =\left\|\alpha e_{1}+\right\| \beta e_{2}+t e_{3}\left\|e_{3}\right\| \text { by }(2) .
\end{aligned}
$$

But

$$
\begin{aligned}
\left\|f_{1}+t f_{2}\right\| & =\left\|f_{1}-t f_{2}\right\| \quad \text { by }(4) \\
& =\left\|\alpha e_{1}+\right\| \beta e_{2}-t e_{3}\left\|e_{3}\right\| \quad \text { as above. }
\end{aligned}
$$

Suppose for purposes of obtaining a contradiction that

$$
\left\|\beta e_{2}+t e_{3}\right\| \neq\left\|\beta e_{2}-t e_{3}\right\| \text { for some } t
$$


Then

$$
\begin{aligned}
\left\|\alpha e_{1}+\right\| \beta e_{2}+t e_{3}\left\|e_{3}\right\| & =\left\|\alpha e_{1}-\right\| \beta e_{2}+t e_{3}\left\|e_{3}\right\| \\
& =\left\|\alpha e_{1}+\right\| \beta e_{2}-t e_{3}\left\|e_{3}\right\|
\end{aligned}
$$

and the convexity of the norm implies that $\left\|\alpha e_{1}+s e_{3}\right\|$ is constant, and hence equal to $\left\|\alpha e_{1}\right\|=|\alpha|$, for $|s| \leqslant \max \left\{\left\|\beta e_{2}+t e_{3}\right\|,\left\|\beta e_{2}-t e_{3}\right\|\right\}=r$. Thus $\left\|\alpha e_{1}+x_{2} e_{2}+x_{3} e_{3}\right\|$ $=|\alpha|$ whenever $\left\|x_{2} e_{2}+x_{3} e_{3}\right\| \leqslant r$.

Thus for $s \leqslant t$ we have

$$
\left\|f_{1}+s e_{3}\right\|=\left\|\alpha e_{1}+\beta e_{2}+s e_{3}\right\|=|\alpha|
$$

and putting $s=0$ we see that $|\alpha|=1$. Now

$$
\begin{aligned}
\left\|f_{1}+s e_{3}\right\| & =\left\|f_{1}+s f_{2}\right\| \quad(\text { by }(4)) \\
& =\left\|\alpha e_{1}+\beta e_{2}+s\left(\gamma e_{1}+\delta e_{2}\right)\right\| \\
& =\left\|(\alpha+s \gamma) e_{1}+(\beta+s \delta) e_{2}\right\| \\
& =|\alpha+s \gamma|\left\|\alpha e_{1}+(\alpha+s \gamma)^{-1}(\beta+s \delta) e_{3}\right\| \\
& =|\alpha+s \gamma| \quad \text { whenever }\left|(\alpha+s \gamma)^{-1}(\beta+s \delta)\right| \leqslant r .
\end{aligned}
$$

The convexity of the norm and (6) show that at least one of $\left\|\beta e_{2}+t e_{3}\right\|$ and $\left\|\beta e_{2}-t e_{3}\right\|$ is greater than $\left\|\beta e_{2}\right\|=|\beta|$. Since $\left|(\alpha+s \gamma)^{-1}(\beta+s \delta)\right|$ is equal to $|\beta|$ when $s=0$, by continuity $\left|(\alpha+s \gamma)^{-1}(\beta+s \delta)\right|<r$ for $s$ near 0 . Using (7) we see that $|\alpha+s \gamma|=|\alpha|=1$ for $s$ near 0 , thus $\gamma=0$.

If necessary taking $f_{1}$ to be $-f$ instead of $f$ we have $f_{1}=e_{1}+\beta e_{2}$ and $f_{2}=$ $\delta e_{2}=\dot{e}_{2}$ without loss of generality. Thus $\left\|f_{1}-\beta e_{2}\right\|=\left\|e_{1}\right\|=1$ so that $\left\|f_{1}+\beta e_{2}\right\|=$ 1 by (4). This means $\left\|e_{1}+2 \beta e_{2}\right\|=1$; so $\left\|e_{1}-2 \beta e_{2}\right\|=1$ by (2), which means $\left\|f_{1}-3 \beta e_{2}\right\|=1$. By induction $\left\|e_{1}+n \beta e_{2}\right\|=1$ for all $n \in \mathrm{N}$, giving $\beta=0$, so that $e$ and $f$ are not linearly independent. This contradiction shows that (6) is false and hence $\left\|\beta e_{2}+t e_{3}\right\|=\left\|\beta e_{2}-t e_{3}\right\|$ for all $t$. This yields (3) and completes the proof.

We will need the following result from [3] or [4].

Proposition 3. Let $X$ be a real three-dimensional Banach lat tice with unit basis $\left\{e_{1}, e_{2}, e_{3}\right\}$ such that $e_{i} \wedge e_{j}=0$ if $i \neq j$. Suppose every subspace which intersects $\left\{e_{1}, e_{2}\right\}$ is the range of a nonexpansive projection on $X$. Then $X$ is isometrically isomorphic to $\ell_{p}(3)$ for some $p \in[1, \infty]$.

Proposition 4. Under our standing assumptions, either $X$ is isometrically isomorphic to $\ell_{p}(3)$ for some $p \in[1, \infty]$ or there is an isometry $R: X \rightarrow X$ such that 
$R e_{3}=e_{3}$ and for some $t>0$ and some odd integer $m>2$, letting $\theta=\pi m^{-1}$ we have $R e_{1}=\cos \theta e_{1}+t^{-1} \sin \theta e_{2}$ and $R e_{2}=\cos \theta e_{2}-t \sin \theta e_{1}$.

Proof: If $f_{1}= \pm e_{2}$ then by Proposition 2, if we order $X$ by the cone generated by $\left\{e_{1}, e_{2}, e_{3}\right\}$ then the hypotheses of Proposition 3 hold and $X$ is isometrically isomorphic to $\ell_{p}(3)$ for some $p \in[1, \infty]$. Thus we assume that $f_{1}=\alpha e_{1}+\beta e_{2} ; \alpha, \beta>0$ without loss of generality (replacing $e_{1}$ or $e_{2}$ by its negative if necessary). Recall that $f_{2}=\gamma e_{1}+\delta e_{2}$. If $\delta=0$ then $f_{2}= \pm e_{1}$ and as above the required conclusion holds by Propositions 2 and 3 , so we take $\delta>0$ without loss of generality, changing the sign of $f_{2}$ if necessary.

Now suppose $\gamma=0$. Then $f_{2}=e_{2}$ so that for all $t,\left\|f_{1}+t e_{2}\right\|=\left\|f_{1}-t e_{2}\right\|$. Thus $\left\|\alpha e_{1}+(\beta+t) e_{2}\right\|=\left\|\alpha e_{1}+(\beta-t) e_{2}\right\|$ and taking $t=n \beta$ we have $\left\|\alpha e_{1}+(n+1) \beta e_{2}\right\|$ $=\left\|\alpha e_{1}+(n-1) \beta e_{2}\right\|$ and for even integers $m$ we get $\left\|\alpha e_{1}+m \beta e_{2}\right\|=\alpha$, so $\beta=0$ giving a contradiction which shows that $\gamma \neq 0$. It will be seen later that $\gamma<0$.

Since $\left\|y_{1} f_{1}+y_{2} f_{2}+x_{3} e_{3}\right\|=\left\|y_{1} f_{1}-y_{2} f_{2}+x_{3} e_{3}\right\|$ for all $y_{1}, y_{2}$ and $x_{3}$, we have $\left\|\left(y_{1} \alpha+y_{2} \gamma\right) e_{1}+\left(y_{1} \beta+y_{2} \delta\right) e_{2}+x_{3} e_{3}\right\|=\left\|\left(y_{1} \alpha-y_{2} \gamma\right) e_{1}+\left(y_{1} \beta-y_{2} \delta\right) e_{2}+x_{3} e_{3}\right\|$. Let $x_{1}=y_{1} \alpha+y_{2} \gamma$ and $x_{2}=y_{1} \beta+y_{2} \delta$ so we have $y_{1}=(\alpha \delta-\beta \gamma)^{-1}\left(\delta x_{1}-\gamma x_{2}\right)$ and $y_{2}=(\alpha \delta-\beta \gamma)^{-1}\left(\alpha x_{2}-\beta x_{1}\right) ; \alpha \delta-\beta \gamma \neq 0$ since $f_{1}$ and $f_{2}$ are independent.

Thus

$$
\begin{aligned}
\cdot\left\|x_{1} e_{1}+x_{2} e_{2}+x_{3} e_{3}\right\|=\| & \left(\alpha \delta x_{1}-\alpha \gamma x_{2}-\alpha \gamma x_{2}+\gamma \beta x_{1}\right)(\alpha \delta-\beta \gamma)^{-1} e_{1} \\
& +\left(\beta \delta x_{1}-\beta \gamma x_{2}-\alpha \delta x_{2}+\beta \delta x_{1}\right)(\alpha \delta-\beta \gamma)^{-1} e_{2}+x_{3} e_{3} \| \\
=\|( & (\alpha \delta+\beta \gamma)(\alpha \delta-\beta \gamma)^{-1} x_{1} e_{1}-2 \alpha \gamma(\alpha \delta-\beta \gamma)^{-1} x_{2} e_{1} \\
& +2 \beta \delta(\alpha \delta-\beta \gamma)^{-1} x_{1} e_{2}-(\alpha \delta+\beta \gamma)(\alpha \delta-\beta \gamma)^{-1} x_{2} e_{2} \\
& +x_{3} e_{3} \| .
\end{aligned}
$$

That means the reflection whose matrix with respect to $\left\{e_{1}, e_{2}, e_{3}\right\}$ is

$$
\left[\begin{array}{ccc}
\frac{\alpha \delta+\beta \gamma}{\alpha \delta-\beta \gamma} & \frac{-2 \alpha \gamma}{\alpha \delta-\beta \gamma} & 0 \\
\frac{2 \beta \delta}{\alpha \delta-\beta \gamma} & \frac{-\alpha \delta-\beta \gamma}{\alpha \delta-\beta \gamma} & 0 \\
0 & 0 & 1
\end{array}\right]
$$

is an isometry. Also by Proposition 2 the reflection whose matrix with respect to the basis $\left\{e_{1}, e_{2}, e_{3}\right\}$ is

$$
\left[\begin{array}{ccc}
1 & 0 & 0 \\
0 & -1 & 0 \\
0 & 0 & 1
\end{array}\right]
$$

is an isometry and thus the composition of these reflections yields an isometry $R$ whose 
matrix with respect to $\left\{e_{1}, e_{2}, e_{3}\right\}$ is

$$
\left[\begin{array}{ccc}
\frac{\alpha \delta+\beta \gamma}{\alpha \delta-\beta \gamma} & \frac{2 \alpha \gamma}{\alpha \delta-\beta \gamma} & 0 \\
\frac{2 \beta \delta}{\alpha \delta-\beta \gamma} & \frac{\alpha \delta+\beta \gamma}{\alpha \delta-\beta \gamma} & 0 \\
0 & 0 & 1
\end{array}\right]
$$

To show that $\gamma<0$ note that $\left\|\gamma \alpha^{-1} f_{1}+f_{2}\right\|=\left\|\gamma \alpha^{-1} f_{1}-f_{2}\right\|$ so that

$$
\left\|\gamma \alpha^{-1}\left(\alpha e_{1}+\beta e_{2}\right)+\gamma e_{1}+\delta e_{2}\right\|=\left\|\gamma \alpha^{-1}\left(\alpha e_{1}+\beta e_{2}\right)-\gamma e_{1}-\delta e_{2}\right\|,
$$

and hence

$$
\left\|2 \gamma e_{1}+\left(\gamma \beta \alpha^{-1}+\delta\right) e_{2}\right\|=\left\|\left(\gamma \beta \alpha^{-1}-\delta\right) e_{2}\right\|=\left|\gamma \beta \alpha^{-1}-\delta\right| .
$$

Now $\left\|2 \gamma e_{1}+\left(\gamma \beta \alpha^{-1}+\delta\right) e_{2}\right\| \geqslant\left|\gamma \beta \alpha^{-1}+\delta\right|$ so that $\delta$ has opposite sign to $\gamma \beta \alpha^{-1}$ and hence to $\gamma$. Thus $\gamma<0$ as claimed, and $-1<(\alpha \delta+\beta \gamma)(\alpha \delta-\beta \gamma)^{-1}<1$. Let $\theta=\cos ^{-1}\left((\alpha \delta+\beta \gamma)(\alpha \delta-\beta \gamma)^{-1}\right)$ and define $t$ by $t \sin \theta=-2 \alpha \gamma(\alpha \delta-\beta \gamma)^{-1}$; since $0<\theta<\pi$ we have $t>0$. The matrix for the isometry $R$ is

$$
\left[\begin{array}{ccc}
\cos \theta & -t \sin \theta & 0 \\
t^{-1} \sin \theta & \cos \theta & 0 \\
0 & 0 & 1
\end{array}\right]
$$

and by an easy induction, for all integers $n$, the matrix for $R^{n}$ is

$$
\left[\begin{array}{ccc}
\cos (n \theta) & -t \sin (n \theta) & 0 \\
t^{-1} \sin (n \theta) & \cos (n \theta) & 0 \\
0 & 0 & 1
\end{array}\right]
$$

Now if $\theta \pi^{-1}$ is irrational then there is a sequence $\left(n_{j}\right)$ of integers such that $\cos \left(n_{j} \theta\right) \rightarrow$ 0 and $\sin \left(n_{j} \theta\right) \rightarrow 1$. Then we have the matrices for $R^{n_{j}}$ converging to

$$
\left[\begin{array}{ccc}
0 & -t & 0 \\
t^{-1} & 0 & 0 \\
0 & 0 & 1
\end{array}\right]
$$

This limit isometry takes $e_{1}$ to $t^{-1} e_{2}$ and hence to $e_{2}$ (and $t=1$ ) so we have nonexpansive projections onto every two-dimensional subspace containing $e_{2}$ and by Proposition 3 the required conclusion holds.

Otherwise there are co-prime integers $k$ and $m$ so that $m \theta=k \pi$. We take integers $i$ and $j$ such that $i k+j m=1$ so that the isometry $(-1)^{j} R^{i}$ has matrix (8) with $\theta=\pi m^{-1}$. We replace $R$ by this isometry and we may assume that $m$ is odd, for otherwise $m=2 b$ and $R^{b}$ has matrix (9) and as above we can apply Proposition 3 .

The next stage is to show that the existence of such an $R$ leads to a Euclidean norm. 
Proposition 5. Suppose under our standing assumptions that there is an isometry $R: X \rightarrow X$ such that $R e_{3}=e_{3}$ and for some $t>0$ and some odd integer $m>2$, letting $\theta=\pi m^{-1}$ we have $R e_{1}=\cos \theta e_{1}+t^{-1} \sin \theta e_{2}$ and $R e_{2}=\cos \theta e_{2}-t \sin \theta e_{1}$. Then $t=1$ and $X$ is isometrically isomorphic to $\ell_{2}(3)$.

Proof: For each $\nu$ there is a nonexpansive projection onto $\operatorname{span}\left\{e_{1}, e_{3}+\nu e_{2}\right\}$ along a vector $p(\nu)$ in $\operatorname{span}\left\{e_{2}, e_{3}\right\}$. We can take $p(\nu)=e_{2}-g(\nu) e_{3}$ unless $p(\nu)$ is in $\mathbf{R e}_{3}$ in which case take $p(\nu)=e_{3}$, in fact we will see this case cannot occur.

For each $\alpha, \nu$ and $s$ we have

$$
\alpha R e_{1}+\nu R e_{2}+e_{3}=(\alpha \cos \theta-\nu t \sin \theta) e_{1}+\left(\nu \cos \theta+\alpha t^{-1} \sin \theta\right) e_{2}+e_{3}
$$

so that if $g\left(\nu \cos \theta+\alpha t^{-1} \sin \theta\right)$ exists we have

$$
\left\|\alpha R e_{1}+\nu R e_{2}+e_{3}\right\| \leqslant\left\|\alpha R e_{1}+\nu R e_{2}+e_{3}+s\left(e_{2}-g\left(\nu \cos \theta+\alpha t^{-1} \sin \theta\right) e_{3}\right)\right\|
$$

and applying the isometry $R^{-1}$ we get

$$
\begin{aligned}
\| \alpha e_{1}+\nu e_{2} & +e_{3}\|\leqslant\| \alpha e_{1}+\nu e_{2}+e_{3}+s\left(R^{-1} e_{2}-g\left(\nu \cos \theta+\alpha t^{-1} \sin \theta\right) e_{3}\right) \| \\
& =\left\|\alpha e_{1}+\nu e_{2}+e_{3}+s\left(\cos \theta e_{2}+t \sin \theta e_{1}-g\left(\nu \cos \theta+\alpha t^{-1} \sin \theta\right) e_{3}\right)\right\|
\end{aligned}
$$

and using $R^{-1}$ instead of $R$, we have

$$
\begin{aligned}
& \left\|\alpha e_{1}+\nu e_{2}+e_{3}\right\| \\
& \quad \leqslant\left\|\alpha e_{1}+\nu e_{2}+e_{3}+s\left(\cos \theta e_{2}-t \sin \theta e_{1}-g\left(\nu \cos \theta-\alpha t^{-1} \sin \theta\right) e_{3}\right)\right\|
\end{aligned}
$$

provided $g\left(\nu \cos \theta-\alpha t^{-1} \sin \theta\right)$ exists.

Now if $x=\alpha e_{1}+\nu e_{2}+e_{3}$ is a smooth point then $J x(p(\nu))=0$ and $J x\left(\cos \theta e_{2}+t \sin \theta e_{1}-g\left(\nu \cos \theta+\alpha t^{-1} \sin \theta\right) e_{3}\right)=0$ and hence we have that $J_{x}\left(\cos \theta e_{2}-t \sin \theta e_{1}-g\left(\nu \cos \theta-\alpha t^{-1} \sin \theta\right) e_{3}\right)=0$ so that the vectors $p(\nu), \cos \theta e_{2}$ $+t \sin \theta e_{1}-g\left(\nu \cos \theta+\alpha t^{-1} \sin \theta\right) e_{3}$ and $\cos \theta e_{2}-t \sin \theta e_{1}-g\left(\nu \cos \theta-\alpha t^{-1} \sin \theta\right) e_{3}$ are linearly dependent. So if $g(\nu)$ exists then

$$
\left|\begin{array}{ccc}
0 & 1 & g(\nu) \\
t \sin \theta & \cos \theta & g\left(\nu \cos \theta+\alpha t^{-1} \sin \theta\right) \\
-t \sin \theta & \cos \theta & g\left(\nu \cos \theta-\alpha t^{-1} \sin \theta\right)
\end{array}\right|=0
$$

That means

$$
2 \cos \theta g(\nu)=g\left(\nu \cos \theta+\alpha t^{-1} \sin \theta\right)+g\left(\nu \cos \theta-\alpha t^{-1} \sin \theta\right)
$$

On the other hand if $g(\nu)$ ever fails to exist then since $0<\cos \theta<1$ we can choose a smooth point $\alpha e_{1}+\nu e_{2}+e_{3}$ so that $p(\nu)=e_{3}$ while $g\left(\nu \cos \theta+\alpha t^{-1} \sin \theta\right)$ 
and $g\left(\nu \cos \theta-\alpha t^{-1} \sin \theta\right)$ both exist. But the vectors $e_{3}, \cos \theta e_{2}+t \sin \theta e_{1}-$ $g\left(\nu \cos \theta+\alpha t^{-1} \sin \theta\right) e_{3}$ and $\cos \theta e_{2}-t \sin \theta e_{1}-g\left(\nu \cos \theta-\alpha t^{-1} \sin \theta\right) e_{3}$ are linearly independent, contradicting the linear dependence noted above. Thus $g(\nu)$ exists for all $\nu$ and (10) holds for almost all $(\alpha, \nu)$ in $\mathbf{R}^{2}$ since almost all points in $X$ are smooth.

Since $g$ is monotone increasing we may assume that $g$ is continuous from the right and it then follows that (10) holds for all $\alpha$ and $\nu$. Putting $\alpha=0$ gives $\cos \theta g(\nu)=$ $g(\cos \theta \nu)$ so we have for all $\nu, y$ in $\mathbf{R}$,

$$
2 g(\nu)=g(\nu+y)+g(\nu-y) \text {. }
$$

It is known (see [0], §72) that the only monotone solutions of (11) are the affine functions $g(\nu)=k \nu+r$ and $r=0$ since $\cos \theta g(\nu)=g(\cos \theta \nu)$. Thus $g(\nu)=k \nu$ for all $\nu$, so for each $\nu$ there is a nonexpansive projection onto $\operatorname{span}\left\{e_{1}, e_{3}+\nu e_{2}\right\}$ along the vector $e_{2}-k \nu e_{3}$. It follows that the convex function $N(x, y)=\left\|x e_{2}+y e_{3}\right\|$ has $\nabla N(x, y) \perp(y,-k x)$ almost everywhere and the solutions of the differential equation

$$
\frac{d y}{d x}=-k \frac{x}{y}
$$

give curves with $N(x, y)$ constant. Thus $y^{2}+k x^{2}=c$ are curves with $N(x, y)$ constant and evaluating at $(1,0)$ and $(0,1)$ we find that if $y^{2}+x^{2}=1$ then $N(x, y)=1$. Thus $\left\|x e_{2}+y e_{3}\right\|=\left(y^{2}+x^{2}\right)^{\frac{1}{2}}$ and so

$$
\begin{aligned}
\left\|x e_{1}+y e_{2}+z e_{3}\right\|= & \left\|x e_{1}+\left(y^{2}+z^{2}\right)^{\frac{1}{2}} e_{3}\right\|=\left\|x R e_{1}+\left(y^{2}+z^{2}\right)^{\frac{1}{2}} e_{3}\right\| \\
= & \left\|x\left(\cos \theta e_{1}+t^{-1} \sin \theta e_{2}\right)+\left(y^{2}+z^{2}\right)^{\frac{1}{2}} e_{3}\right\| \\
= & \left\|x \cos \theta e_{1}+\left(t^{-2} \sin ^{2} \theta x^{2}+y^{2}+z^{2}\right)^{\frac{1}{2}} e_{3}\right\| \\
= & \| x \cos ^{2} \theta e_{1} \\
& \quad+\left(\left(1+\cos ^{2} \theta\right) t^{-2} \sin ^{2} \theta x^{2}+y^{2}+z^{2}\right)^{\frac{1}{2}} e_{3} \| \\
= & \| x \cos ^{n} \theta e_{1} \\
& \quad+\left(\left(1+\cos ^{2} \theta+\cdots+\cos ^{2 n-2} \theta\right) t^{-2} \sin ^{2} \theta x^{2}+y^{2}+z^{2}\right)^{\frac{1}{2}} e_{3} \|
\end{aligned}
$$

by an easy induction, so that letting $n \rightarrow \infty$ we therefore have $\left\|x e_{1}+y \epsilon_{2}+z e_{3}\right\|=$ $\left\|\left(t^{-2} x^{2}+y^{2}+z^{2}\right)^{\frac{1}{2}} e_{3}\right\|$. Evaluating at $e_{1}$ gives $t=1$ and $\left\|x \epsilon_{1}+y \epsilon_{2}+z e_{3}\right\|=$ $\left(x^{2}+y^{2}+z^{2}\right)^{\frac{1}{2}}$ as required.

Putting these propositions together we have proved the following result. 
Theorem A. Let $X$ be a 3-dimensional normed space with linearly independent smooth points $e$ and $f$ such that every 2-dimensional subspace which intersects $\{e, f\}$ is the range of a nonexpansive projection. Then there is $p \in(1, \infty]$ such that $X$ is isometrically isomorphic to $\ell_{p}(3)$.

Proof: Using Propositions 4 and 5 we see that $X$ is isomorphic to $\ell_{p}(3)$ for some $p \in[1, \infty]$. But $\ell_{1}(3)$ does not have any smooth points $e$ such that every 2 -dimensional subspace containing $e$ is the range of a nonexpansive projection.

Corollary. Let $X$ be a 3 -dimensional normed space with basis $\left\{e_{1}, e_{2}, e_{3}\right\}$ of smooth points such that every 2-dimensional subspace which intersects $\left\{e_{1}, e_{2}, e_{2}\right\}$ is the range of a nonexpansive projection. Then there is $p \in(1, \infty]$ such that $X$ is isometrically isomorphic to $\ell_{p}(3)$.

\section{SPACES OF higher Dimension}

We first need to record which points in $\ell_{p}(n)$ have the property we are interested in.

Proposition 6. If $e \in \ell_{p}(n), n>2, p \neq 2$ is a smooth point such that every two-dimensional subspace containing $e$ is the range of a nonexpansive projection then e has exactly one nonzero coordinate.

Proof: Let $e=\left(1, \alpha_{2}, \alpha_{3}, \ldots, \alpha_{n}\right)$ where $\alpha_{2} \neq 0$ and $\left|\alpha_{i}\right| \leqslant 1$ for each $i$. Let $x=(0,1,-1,0, \ldots, 0)$ if $\alpha_{2} . \alpha_{3}>0$ and $x=(0,1,1,0, \ldots, 0)$ otherwise. Then $\operatorname{span}\{e, x\}$ is not the range of a nonexpansive projection. For $p$ finite this is a consequence of [7], Theorem 2.a.4. If $p=\infty$ then smoothness at $e$ implies that $\left|\alpha_{i}\right| \neq 1$; then suppose that $P$ is a nonexpansive projection onto $\operatorname{span}\{e, x\}$. Since, for $j=1,2$ and $3, \operatorname{span}\{e, x\}$ intersects the interior of the face of the unit sphere in $\ell_{\infty}(n)$ on which the $j^{\text {th }}$ coordinate is 1 , we have $\operatorname{dim} P^{-1}(0) \leqslant n-3$. But that means the range of $P$ is at least 3 -dimensional, giving a contradiction.

The smoothness at $e$ is needed in the case of $\ell_{\infty}(n)$ because for example every 2-dimensional subspace containing $e=(1,1, \ldots, 1)$ is the range of a nonexpansive projection.

Theorem B. Let $X$ be a $n$-dimensional normed space and let $\left\{e_{2}, e_{3}, \ldots, e_{n}\right\}$ be a linearly independent set of smooth points in $X$ such that every 2-dimensional subspace intersecting $\left\{e_{2}, e_{3}, \ldots, e_{n}\right\}$ is the range of a nonexpansive projection. Then $X$ is isometrically isomorphic to $\ell_{p}(n)$ for some $p \in(1, \infty)$.

Proof: This is true for $n=3$ by Theorem A. Let $k>3$ and assume that it is true for $n=k-1$. Suppose $\left\{e_{2}, e_{3}, \ldots, e_{k}\right\}$ is a linearly independent set of smooth points in $X$ such that every 2-dimensional subspace intersecting $\left\{e_{2}, e_{3}, \ldots, e_{k}\right\}$ is a the range 
of a nonexpansive projection. We choose $e_{1}$ such that $e_{1} \notin \operatorname{span}\left\{e_{2}, e_{3}, \ldots, e_{k}\right\}$ and $J e_{i}\left(e_{1}\right)=0$ for $2 \leqslant i \leqslant k$ and we also suppose that $\left\|e_{i}\right\|=1$ for $1 \leqslant i \leqslant k$.

Let $x_{i}$ be scalars, $1 \leqslant i \leqslant k$, with $x_{1} \neq 0$. By our inductive hypothesis there are $p, q$ and $r$ in $(1, \infty)$ such that

$\operatorname{span}\left\{e_{2}, e_{3}, \ldots, e_{k}\right\}$ is isometrically isomorphic to $\ell_{p}(k-1)$, $\operatorname{span}\left\{e_{1}, e_{2}, \ldots, e_{k-1}\right\}$ is isometrically isomorphic to $\ell_{q}(k-1)$, and $\operatorname{span}\left\{x_{1} e_{1}+x_{2} e_{2}, e_{3}, \ldots, e_{k}\right\}$ is isometrically isomorphic to $\ell_{r}(k-1)$.

Now $\operatorname{span}\left\{e_{2}, e_{3}, \ldots, e_{k-1}\right\}$ is isometrically isomorphic to $\ell_{p}(k-2)$ by Proposition 6 if $p \neq 2$ and by subspaces of Euclidean spaces being isometrically isomorphic to Euclidean spaces if $p=2$. Similarly $\operatorname{span}\left\{e_{2}, e_{3}, \ldots, e_{k-1}\right\}$ is isometrically isomorphic to $\ell_{q}(k-2)$, so we have $p=q$. Considering $\operatorname{span}\left\{e_{3}, \ldots, e_{k}\right\}$ we see similarly that $p=r$.

If $p \neq 2$ then Proposition 6 and our choice of $e_{1}$ show that $e_{i}$ is orthogonal to $x_{1} e_{1}+x_{2} e_{2}$ for $3 \leqslant i \leqslant k$, giving

$$
\left\|\sum_{i=1}^{k} x_{i} e_{i}\right\|=\left\|\left(\left\|x_{1} e_{1}+x_{2} e_{2}\right\|, x_{3}, \ldots, x_{k}\right)\right\|_{p}
$$

and similarly $\left\|x_{1} e_{1}+x_{2} e_{2}\right\|=\left(x_{1}, x_{2}\right)_{p}$ so that, as required

$$
\left\|\sum_{i=1}^{k} x_{i} e_{i}\right\|=\left\|\left(x_{1}, x_{2}, \ldots, x_{k}\right)\right\|_{p}
$$

If $p=2$ then $J e_{2}\left(e_{1}\right)=0$ implies that $\left\|x_{1} e_{1}+x_{2} e_{2}\right\|^{2}=x_{1}^{2}+x_{2}^{2}$. Since $\operatorname{span}\left\{x_{1} e_{1}+x_{2} e_{2}, e_{3}, \ldots, e_{k}\right\}$ is isometrically isomorphic to $\ell_{2}(k-1)$ we have

$$
\begin{aligned}
\left\|\sum_{i=1}^{k} x_{i} e_{i}\right\|^{2} & =\left\langle\sum_{i=1}^{k} x_{i} e_{i}, \sum_{i=1}^{k} x_{i} e_{i}\right\rangle \\
& =\left\|x_{1} e_{1}+x_{2} e_{2}\right\|^{2}+\left\langle\sum_{i=3}^{k} x_{i} e_{i}, 2 x_{1} e_{1}+2 x_{2} e_{2}+\sum_{i=3}^{k} x_{i} e_{i}\right\rangle \\
& =\sum_{i=1}^{k} x_{i}^{2}+2 \sum_{i=3}^{k} x_{i} J e_{i}\left(x_{1} e_{1}+x_{2} \epsilon_{2}\right)+2 \sum_{i=3}^{k-1} \sum_{j=i+1}^{k} x_{i} x_{j} J \epsilon_{j}\left(c_{i}\right) \\
& =\sum_{i=1}^{k} x_{i}^{2}+2 \sum_{j=2}^{k-1} \sum_{i=j+1}^{k} J e_{i}\left(e_{j}\right) x_{i} x_{j}
\end{aligned}
$$


which shows that the norm of $\operatorname{span}\left\{e_{1}, e_{2}, \ldots, e_{k}\right\}$ is Euclidean, as required to complete the proof by induction.

Note that the corollary to Theorem A does not suffice to prove the corresponding weaker form of Theorem $\mathrm{B}$ with $\left\{e_{2}, e_{3}, \ldots, e_{n}\right\}$ replaced by $\left\{e_{1}, e_{2}, e_{3}, \ldots, e_{n}\right\}$. This theorem extends to infinite dimensional spaces as follows.

Theorem C. Let $E$ be a Banach space of dimension at least 3 over $\mathbf{R}$ and let $\left\{e_{i}: i \in I\right\}$ be a linearly independent set of smooth points with $\operatorname{span}\left\{e_{i}: i \in I\right\}$ dense in $E$. Suppose that every two-dimensional subspace intersecting $\left\{e_{i}: i \in I\right\}$ is the range of a nonexpansive projection. Then either

(a) $E$ is isometrically isomorphic to $c_{0}(I)$ or to $\ell_{p}(I)$ for some $p \neq 2$, in such a way that each $e_{i}$ corresponds to an element of the canonical basis or

(b) $E$ is isometrically isomorphic to a Hilbert space.

Proof: If $I$ is finite then this follows from Theorem B and Proposition 6. Otherwise there is $p$ such that for each finite subset $F$ of $I, \operatorname{span}\left\{e_{i}: i \in F\right\}$ is isometrically isomorphic to $\ell_{p}(F)$, with each $e_{i}, i \in F$, corresponding to an element of the canonical basis in $\ell_{p}(F)$ if $p \neq 2$. For $p=\infty$ it follows that $E$ is isometrically isomorphic to $c_{0}(I)$, while for finite $p \neq 2$ it follows that $E$ is isometrically isomorphic to $\ell_{p}(I)$, in both cases the elements $e_{i}$ corresponding to canonical basis elements.

In the case $p=2$ let $x, y \in E$ and let $x_{n}, y_{n}$ be elements of $\operatorname{span}\left\{e_{i}: i \in I\right\}$ such that $\left\|x-x_{n}\right\|$ and $\left\|y-y_{n}\right\|$ are less than $n^{-1}$. Then

$$
\left\langle x_{n}, y_{n}\right\rangle=4^{-1}\left(\left\|x_{n}+y_{n}\right\|^{2}-\left\|x_{n}-y_{n}\right\|^{2}\right) \rightarrow 4^{-1}\left(\|x+y\|^{2}-\|x-y\|^{2}\right)
$$

so we define $\langle x, y\rangle$ to be

$$
4^{-1}\left(\|x+y\|^{2}-\|x-y\|^{2}\right)=\lim \left\langle x_{n}, y_{n}\right\rangle .
$$

Then $\langle$,$\rangle is a bilinear form such that \|x\|^{2}=\langle x, x\rangle$ and $E$ is a Hilbert space.

Remark. The difference between this result and our previous work [3], [4] and [5] is that the Banach space does not need to be a lattice and the points $e_{i}$ do not need to be orthogonal. We do require the norm to be smooth at the points $\epsilon_{i}$; without this some other spaces satisfy our standard hypotheses.

The condition that $\operatorname{span}\left\{e_{i}: i \in I\right\}$ is dense in $E$ can be weakened to $\operatorname{span}\left\{e_{i}\right.$ : $i \in I\}$ being dense in some hyperplane in $E$. A similar modification is possible in our final result which is a characterisation of real Hilbert spaces. 
Theorem D. Let $E$ be a real Banach space of dimension at least 3 . Then $E$ is a Hilbert space if and only if there is a linearly independent set $A$ of smooth points in $E$ such that the linear span of $A$ is dense in $E$, every 2-dimensional subspace intersecting $A$ is the range of a nonexpansive projection and $\|x+t y\|<\|x\|$ for some distinct $x, y \in A$ and $t \in \mathbf{R}$.

Proof: In $c_{0}$ and $\ell_{p}, p \neq 2$, the elements of $A$ must be mutually orthogonal.

\section{REFERENCES}

[1] T. Ando, 'Banachverbande und positive projection', Math. $Z .109$ (1969), 121-130.

[2] W. Blaschke, 'Raumliche Variationsprobleme mit symmetrischer Transversalitatsbedingung', Leipziger Berichte, Math-phys. Klasse 68 (1916), 50-55.

[3] Bruce Calvert and Simon Fitzpatrick, 'A Banach lattice characterisation of co and $\ell_{p}$ ', (submitted).

[4] Bruce Calvert and Simon Fitzpatrick, 'Consequences of the duality map taking planes to planes' (to appear), in Proceedings of the VII International Conference on Nonlinear Analysis and Applications, ed. V. Lakshmikantham to appear.

[5] Bruce Calvert and Simon Fitzpatrick, 'Characterising $\ell_{p}$ and $c_{0}$ by projections onto hyperplanes', Boll. Un. Math. Ital. (to appear).

[6] H.E. Lacy, The Isometric Theory of Classical Banach Spaces (Springer-Verlag, Berlin, 1974).

[7] J. Lindenstrauss and L. Tzafriri, Classical Banach Spaces I (Springer-Verlag, Berlin, 1977).

[8] J. Lindenstrauss and L. Tzafriri, Classical Banach Spaces II (Springer-Verlag, Berlin, 1979).

[9] A. Wayne Roberts and Dale E. Varberg, Convex Functions (Academic Press, New York, 1973).

Dr. B. Calvert

Department of Mathematics and Statistics

The University of Auckland

Private Bag

A uckland

New Zealand 